\title{
Hyers-Ulam stability of iterative equations in Banach spaces
}

\author{
Xi Wang \\ Faculty of Mathematics and Statistics, Changchun University of Technology, Changchun 130012 China \\ e-mail: wangxi@ccut.edu.cn
}

Received 20 Aug 2020

Accepted 23 Dec 2020

\begin{abstract}
In the present paper, we discuss the Hyers-Ulam stability of the equation $\mathscr{G} f(f(x))=\mathscr{F} f(x)$ in a Banach space. By constructing a uniformly convergent sequence of functions, we prove that if one can find an approximate solution of such an equation, then there must be a unique solution close to the approximate solution.
\end{abstract}

KEYWORDS: Hyers-Ulam stability, iterative equation, Banach space

MSC2010: 39B82 39B12 47H99

\section{INTRODUCTION}

The study of stability of functional equations originated from a question of Ulam [1] concerning the stability of group homomorphisms. The question is stated as follows.

Given a group $G_{1}$ and a metric group $G_{2}$ with metric $\rho(\cdot, \cdot)$. Given $\epsilon>0$, does there exist a $\delta>0$ such that if $f: G_{1} \rightarrow G_{2}$ satisfies $\rho(f(x y), f(x) f(y))<\delta$ for all $x, y \in G_{1}$, then a homomorphism $h: G_{1} \rightarrow G_{2}$ exists with $\rho(f(x), h(x))<\epsilon$ for all $x \in G_{1}$ ?

The problem for Cauchy equation in Banach space was solved by Hyers [2] in 1941.

In this paper, we consider the problem for iterative equation in Banach space. Let $C(X, X)$ be the set of all continuous self-mappings on a topological space $X$. For any $f \in C(X, X)$, let $f^{m}$ denote the $m$-th iterate of $f$, i.e., $f^{m}=f \circ f^{m-1}, f^{0}=$ id, $m=1,2, \ldots$. Equations having iteration as their main operation, that is, including iterates of the unknown mapping, are called iterative equations. It is one of the most interesting classes of functional equations $[3,4]$, because it includes the problem of iterative roots [3], i.e. finding some $f \in C(X, X)$ such that $f^{n}$ is identical to a given $F \in C(X, X)$.

As a natural generalization of the problem of iterative roots, iterative equations of the following form

$$
\lambda_{1} f(x)+\lambda_{2} f^{2}(x)+\cdots+\lambda_{n} f^{n}(x)=F(x)
$$

for $x \in I=[a, b]$, are known as polynomial-like iterative equations. Here $n \geqslant 2$ is an integer, $\lambda_{i} \in \mathbb{R}$, $i=1,2, \ldots, n, F: I \rightarrow \mathbb{R}$ is a given mapping and $f:$
$I \rightarrow I$ is unknown. As mentioned in [5], polynomiallike iterative equations are important not only in the study of functional equations but also in the study of dynamical systems. For instance, such equations are encountered in the discussion on transversal homoclinic intersection for diffeomorphisms [6], normal form of dynamical systems [7], and dynamics of a quadratic mapping [8]. Some problems of invariant curves for dynamical systems also lead to such iterative equations [9].

Note that the polynomial-like iterative equation

$$
\sum_{i} \alpha_{i} f^{i}=F,
$$

where $\sum_{i} \alpha_{i}=1$, is a particular case of (1).

A successful approach to deal with this equation with the use of a fixed point method was introduced by Zhang [10] in 1987. The idea of the proof of the main theorem in [10] is the construction of a structural operator. Following this idea, we construct an operator to prove the main theorem in this work.

In [11], a higher dimension case, where the domain of $F$ is a compact convex subset of $\mathbb{R}^{n}$, was treated. Tabor [12] generalized the domain in [11] to an arbitrary closed subset of a Banach space. In this paper, we will allow operator coefficients, and use the Zhang's method [10] to discuss the following equation.

$$
\mathscr{G} f(f(x))=\mathscr{F} f(x) \quad \text { for } x \in V .
$$

In this work, we will consider the Hyers-Ulam stability of (2). As an example, we will discuss 
the stability of a polynomial-like iterative equation. For more details in Hyers-Ulam stability of iterative equations, please refer to [13].

\section{THE MAIN RESULT}

As our results are based on the Banach contraction principle, we first need to describe the space on which our results will take place.

Let $X$ be a Banach space. For a function $f: S \rightarrow$ $X$, where $S$ is a subset of $X$, we put

$$
\begin{aligned}
\|f\|_{\text {sup }} & :=\sup _{s \in S}\|f(s)\|, \\
k(f) & :=\operatorname{Lip}(f-\mathrm{id}),
\end{aligned}
$$

where Lip denotes the Lipschitz constant. One can easily notice that $\|\cdot\|_{\text {sup }}$ defines a metric (which can possibly attain $+\infty$ ) by the formula $d_{\text {sup }}(f, g):=$ $\|f-g\|_{\text {sup }}$.

Let $V$ be a closed subset of $X$. We define

$$
\begin{aligned}
\mathscr{K}(V) & =\left\{f: V \rightarrow X|f|_{\partial V}=\left.\mathrm{id}\right|_{\partial V},\|f-\mathrm{id}\|_{\text {sup }}<\infty\right\}, \\
\mathscr{K}_{m}(V) & =\{f \in \mathscr{K}(V) \mid k(f) \leqslant m\} \quad \text { for } m \geqslant 0 .
\end{aligned}
$$

The space $\left(\mathscr{K}(V), d_{\text {sup }}\right)$ is a complete metric space, and $\mathscr{K}_{m}(V)$ is a closed subset of $\mathscr{K}(V)$.

Now we are ready to present our main tool to be used further on. We first recall some lemmas.

Lemma 1 ([12]) Let $f, g, f_{1}, g_{2} \in \mathscr{K}(V), f_{1}(V) \subset V$ and $g_{1}(V) \subset V$.

(i) If $\operatorname{Lip}(f)<\infty$, then

$$
\begin{aligned}
\left\|f \circ f_{1}-g \circ g_{1}\right\|_{\text {sup }} \\
\quad \leqslant \operatorname{Lip}(f)\left\|f_{1}-g_{1}\right\|_{\text {sup }}+\|f-g\|_{\text {sup }} .
\end{aligned}
$$

(ii) We assume that $f$ and $g$ are invertible and $\operatorname{Lip}\left(f^{-1}\right)<\infty$. Then

$$
\left\|f^{-1}-g^{-1}\right\|_{\text {sup }} \leqslant \operatorname{Lip}\left(f^{-1}\right)\|f-g\|_{\text {sup }} .
$$

\section{Lemma 2 ([12])}

(i) Let $f, g \in \mathscr{K}(V)$, and $g(V) \subset V$. Then $f \circ g \in$ $\mathscr{K}(V)$ and

$$
k(f \circ g) \leqslant(1+k(f))(1+k(g))-1 .
$$

(ii) Let $f \in \mathscr{K}(V)$ be such that $k(f)<1$. Then $f(V) \subset V$.

(iii) Let $f \in \mathscr{K}(V)$ be such that $k(f)<1$. Then $f^{-1} \in$ $\mathscr{K}(V)$ and

$$
k\left(f^{-1}\right) \leqslant \frac{1}{1-k(f)}-1 .
$$

Lemma 3 ([12]) Let $f \in \mathscr{K}(V)$ be such that $k(f)<1$. Then $f^{-1} \in \mathscr{K}(V)$ and

$$
\operatorname{Lip}\left(f^{-1}\right) \leqslant \frac{1}{1-k(f)} .
$$

Now we present the main result of this work.

Theorem 1 Let $m \in(0,1)$ be given. Let $\mathscr{F}, \mathscr{G}$ : $\mathscr{K}_{m}(V) \rightarrow \mathscr{K}(V)$ be fixed. Let

$$
\begin{aligned}
& k_{\mathscr{F}}:=\sup \left\{k(\mathscr{F} f): f \in \mathscr{K}_{m}(V)\right\} \text { and } \\
& k_{\mathscr{G}}:=\sup \left\{k(\mathscr{G} f): f \in \mathscr{K}_{m}(V)\right\} .
\end{aligned}
$$

Suppose that

(P1) $\left(1+k_{\mathscr{F}}\right) /\left(1-k_{\mathscr{G}}\right) \leqslant 1+m$,

(P2) $\operatorname{Lip}(\mathscr{F})+\operatorname{Lip}(\mathscr{G})<1-k_{g}$.

For any $\delta>0$, if there is a $g \in K_{m}(V)$ such that

$$
\|\mathscr{G}(g) \circ g-\mathscr{F}(g)\|_{\text {sup }} \leqslant \delta,
$$

then (2) has a unique solution $f$ in $\mathscr{K}_{m}(V)$, with

$$
\|f-g\|_{\text {sup }} \leqslant \frac{1}{1-k_{\mathscr{G}}-\operatorname{Lip}(\mathscr{F})-\operatorname{Lip}(\mathscr{G})} \delta .
$$

Proof: By condition (P2), $k(\mathscr{G}(f))<1$ for $f \in$ $\mathscr{K}_{m}(V)$. We construct a sequence $\left\{f_{s}\right\} \subset \mathscr{K}(V)$ of functions as follows. Take $f_{0}=g$ first, then define

$f_{s}(x)=\left(\left(\mathscr{G}\left(f_{s-1}\right)\right)^{-1} \circ \mathscr{F}\left(f_{s-1}\right)\right)(x), \quad \forall x \in V, s \in \mathbb{N}$.

We claim that $f_{s} \in \mathscr{K}_{m}(V)$ for any positive integer $s$, and will prove by induction.

First $f_{0}=g \in \mathscr{K}_{m}(V)$, we assume this is true for an integer $s$, then by the first and the third inequalities in Lemma 2, we have

$$
\begin{aligned}
k\left(f_{s+1}\right) & =k\left(\left(\mathscr{G}\left(f_{s}\right)\right)^{-1} \circ \mathscr{F}\left(f_{s}\right)\right) \\
& \leqslant\left(1+k_{\mathscr{G}-1}\right)\left(1+k_{\mathscr{F}}\right)-1 \\
& \leqslant \frac{1+k_{\mathscr{F}}}{1-k_{\mathscr{G}}}-1 \leqslant m,
\end{aligned}
$$

which means that $f_{s+1} \in \mathscr{K}_{m}(V)$.

Subsequently, we show that the inequality

$$
\left\|f_{s}-f_{s-1}\right\|_{\text {sup }} \leqslant \frac{1}{1-k_{\mathscr{G}}} M^{s-1} \delta
$$

holds for $s=0,1,2, \ldots$, where $M=\frac{\operatorname{Lip}(\mathscr{F})+\operatorname{Lip}(\mathscr{G})}{1-k_{\mathscr{G}}}$. We prove this inequality by induction as well. First,

$$
\begin{aligned}
\left\|f_{1}-f_{0}\right\|_{\text {sup }} & =\left\|(\mathscr{G}(g))^{-1} \circ \mathscr{F}(g)-g\right\|_{\text {sup }} \\
& \leqslant \operatorname{Lip}\left((\mathscr{G}(g))^{-1}\right)\|\mathscr{F}(g)-\mathscr{G}(g) \circ g\|_{\text {sup }} \\
& \leqslant \operatorname{Lip}\left((\mathscr{G}(g))^{-1}\right) \cdot \delta \\
& \leqslant \frac{1}{1-k_{\mathscr{G}}} \delta .
\end{aligned}
$$


Suppose that (4) holds for an integer $s$. Then, for the integer $s+1$, by Lemma 1 and Lemma 3, we have

$$
\begin{aligned}
&\left\|f_{s+1}-f_{s}\right\|_{\text {sup }} \\
&=\left\|\left(\mathscr{G}\left(f_{s}\right)\right)^{-1} \circ \mathscr{F}\left(f_{s}\right)-\left(\mathscr{G}\left(f_{s-1}\right)\right)^{-1} \circ \mathscr{F}\left(f_{s-1}\right)\right\|_{\text {sup }} \\
& \leqslant \frac{1}{1-k_{\mathscr{G}}}\left\|\mathscr{F}\left(f_{s}\right)-\mathscr{F}\left(f_{s-1}\right)\right\|_{\text {sup }} \\
& \quad+\left\|\left(\mathscr{G}\left(f_{s}\right)\right)^{-1}-\left(\mathscr{G}\left(f_{s-1}\right)\right)^{-1}\right\|_{\text {sup }} \\
& \leqslant \frac{\operatorname{Lip}(\mathscr{F})+\operatorname{Lip}(\mathscr{G})}{1-k_{\mathscr{G}}}\left\|f_{s}-f_{s-1}\right\|_{\text {sup }} \leqslant \frac{1}{1-k_{\mathscr{G}}} M^{s} \delta .
\end{aligned}
$$

We claim that $\left\{f_{s}\right\}_{s=0}^{\infty}$ is a Cauchy sequence, since for any two positive integers $s$ and $t$ with $s>t$, we have

$$
\begin{aligned}
\left\|f_{s}-f_{t}\right\|_{\text {sup }} & \leqslant\left\|f_{s}-f_{s-1}\right\|_{\text {sup }}+\cdots+\left\|f_{t+1}-f_{t}\right\|_{\text {sup }} \\
& \leqslant \frac{1}{1-k_{\mathscr{G}}}\left(M^{s-1} \delta+M^{s-2} \delta+\cdots+M^{t} \delta\right) \\
& =\frac{\delta}{1-k_{\mathscr{G}}} \cdot \frac{M^{t}-M^{s}}{1-M} .
\end{aligned}
$$

By conditions (P1) and (P2), $0<M<1$, it follows that

$$
\left\|f_{s}-f_{t}\right\|_{\text {sup }} \rightarrow 0, \quad \text { as } \quad s>t \rightarrow \infty .
$$

As $\mathscr{K}_{m}(V)$ is a closed subset of $\mathscr{K}(V),\left\{f_{s}\right\}_{s=0}^{\infty}$ converges uniformly in $\mathscr{K}_{m}(V)$. Let

$$
\lim _{k \rightarrow \infty} f_{k}=f .
$$

Clearly, $f \in \mathscr{K}_{m}(V)$. From

$$
\begin{aligned}
& \|\mathscr{F}(f)-\mathscr{G}(f) \circ f\|_{\text {sup }}=\lim _{s \rightarrow \infty}\left\|\mathscr{F}\left(f_{s}\right)-\mathscr{G}\left(f_{s}\right) \circ f_{s}\right\|_{\text {sup }} \\
& \quad=\lim _{s \rightarrow \infty}\left\|\mathscr{G}\left(f_{s}\right) \circ\left(\mathscr{G}\left(f_{s}\right)\right)^{-1} \circ \mathscr{F}\left(f_{s}\right)-\mathscr{G}\left(f_{s}\right) \circ f_{s}\right\|_{\text {sup }} \\
& \quad \leqslant \lim _{s \rightarrow \infty} \operatorname{Lip}(\mathscr{G}) \cdot \|\left(\mathscr{G}\left(f_{s}\right)^{-1} \circ \mathscr{F}\left(f_{s}\right)-f_{s} \|_{\text {sup }}\right. \\
& \quad \leqslant \lim _{s \rightarrow \infty} \operatorname{Lip}(\mathscr{G}) \cdot M^{s} \delta=0
\end{aligned}
$$

we know that $f$ is a solution of (2). Furthermore, as $M=\frac{\operatorname{Lip}(\mathscr{F})+\operatorname{Lip}(\mathscr{G})}{1-k_{\mathscr{G}}}$, we have

$$
\begin{aligned}
\|f-g\|_{\text {sup }} & =\lim _{s \rightarrow \infty}\left\|f_{s}-f_{0}\right\|_{\text {sup }} \\
& \leqslant \lim _{s \rightarrow \infty}\left\{\left\|f_{s}-f_{s-1}\right\|_{\text {sup }}+\cdots+\left\|f_{1}-f_{0}\right\|_{\text {sup }}\right\} \\
& \leqslant \lim _{s \rightarrow \infty} \frac{1}{1-k_{\mathscr{G}}}\left\{M^{s-1}+M^{s-2}+\cdots+1\right\} \delta \\
& =\frac{1}{\left(1-k_{\mathscr{G}}\right)(1-M)} \delta \\
& =\frac{1}{1-k_{\mathscr{G}}-\operatorname{Lip}(\mathscr{F})-\operatorname{Lip}(\mathscr{G})} \delta .
\end{aligned}
$$

This proves (3) in Theorem 1.

Concerning uniqueness, we assume that there is another solution $\phi \in \mathscr{K}_{m}(V)$ for (2), such that $\|\phi-g\| \leqslant \varepsilon$, where $\varepsilon>0$ only depends on $\delta$. By Lemma 1 , it follows that

$$
\begin{gathered}
\|f-\phi\|_{\text {sup }}=\left\|(\mathscr{G}(f))^{-1} \circ \mathscr{F}(f)-(\mathscr{G}(\phi))^{-1} \circ \mathscr{F}(\phi)\right\|_{\text {sup }} \\
\leqslant \frac{1}{1-k_{\mathscr{G}}}\|\mathscr{F} f-\mathscr{F} \phi\|_{\text {sup }}+\left\|(\mathscr{G} f)^{-1}-(\mathscr{G} \phi)^{-1}\right\|_{\text {sup }} \\
\leqslant \frac{\operatorname{Lip}(\mathscr{F})+\operatorname{Lip}(\mathscr{G})}{1-k_{\mathscr{G}}}\|f-\phi\|_{\text {sup }}=M\|f-\phi\|_{\text {sup }},
\end{gathered}
$$

that implies

$$
(1-M)\|f-\phi\|_{\text {sup }} \leqslant 0 .
$$

However, $M<1$, this implies that $\|f-\phi\|_{\text {sup }}=0$, i.e. $f=\phi$.

Corollary 1 Let $m \in(0,1)$ be given. Let $F \in \mathscr{K}_{m}(V)$ and $\mathscr{G}: \mathscr{K}_{m}(V) \rightarrow \mathscr{K}(V)$ be fixed, and

$$
k_{\mathscr{G}}:=\sup \left\{k(\mathscr{G} f): f \in \mathscr{K}_{m}(V)\right\} .
$$

We assume that

$$
\frac{1+k(F)}{1+m} \leqslant 1-k_{\mathscr{G}} \text { and } \operatorname{Lip}(\mathscr{G})<1-k_{\mathscr{G}} .
$$

For any $\delta>0$, if there is a $g \in K_{m}(V)$ such that

$$
\|\mathscr{G}(g) \circ g-F\|_{\text {sup }} \leqslant \delta,
$$

then the equation

$$
(\mathscr{G}(f) \circ f)(x)=F(x) \text { for } \quad x \in V
$$

has a unique solution $f$ in $\mathscr{K}_{m}(V)$ with

$$
\|f-g\|_{\text {sup }} \leqslant \frac{1}{1-k_{\mathscr{G}}-\operatorname{Lip}(\mathscr{G})} \delta .
$$

\section{STABILITY OF SERIES-LIKE ITERATIVE EQUATION}

By $\mathscr{L}(V)$ we denote the Banach space of bounded linear operators on $X$ with the operator norm. We assume that a sequence $\left\{L_{i}\right\}_{i=0}^{\infty} \subset \mathscr{L}(X)$ is given such that

$$
\sum_{i=1}^{\infty} L_{i}=\mathrm{id} \text { and } \sum_{i=1}^{\infty} L_{i} \circ f^{i}=F,
$$

for $f, F \in \mathscr{K}_{m}(V), L_{i} \in \mathscr{L}(X), i \in \mathbb{N}$.

Define, for $m \in(0,1)$,

$$
W(m):=\sum_{i=1}^{\infty}\left\|L_{i}\right\| \cdot\left((1+m)^{i-1}-1\right) .
$$

The proof of the following theorem is similar to that of Theorem 2 in [12]. 
Theorem 2 Let $m \in(0,1)$. Let $V$ be a nonempty closed subset of a Banach spaces $X$, and let $F \in \mathscr{K}(V)$. If

$$
(1+m)(1-W(m))>1+k(F)
$$

then for any $g \in \mathscr{K}_{m}(V)$ with

$$
\left\|F-\sum_{i=1}^{\infty} L_{i} g^{i}\right\|_{\text {sup }} \leqslant \delta
$$

where $\delta$ is a constant, there exists a unique solution $f \in \mathscr{K}_{m}(V)$ of (5) such that

$$
\|f-g\|_{\text {sup }} \leqslant \frac{m}{m-W(m)-m W(m)} \delta .
$$

Proof: Put

$$
\mathscr{P} \varphi:=\sum_{i=1}^{\infty} L_{i} \varphi^{i-1} .
$$

First, we show that for each $\varphi \in \mathscr{K}_{m}(V)$ the function $\mathscr{P} \varphi$ is well defined, that is to say we need to show that $\limsup _{k, n \rightarrow \infty} \sum_{k<i<n}\left\|L_{i} \varphi^{i-1}(x)\right\|=0$.

For any two functions $\varphi, \psi \in \mathscr{K}_{m}(V)$, by Lemma 1, we have

$$
\begin{aligned}
& \sum_{i=1}^{\infty}\left\|L_{i}\left(\varphi^{i-1}-\psi^{i-1}\right)\right\|_{\text {sup }} \\
& \quad \leqslant \sum_{i=1}^{\infty}\left\|L_{i}\right\| \frac{(1+m)^{i-1}-1}{1+m-1}\|\varphi-\psi\|_{\text {sup }} \\
& \quad=\frac{W(m)}{m}\|\varphi-\psi\|_{\text {sup }} .
\end{aligned}
$$

From the first inequality given in the assumption of this theorem, it follows that $\sum_{i=1}^{\infty}\left\|L_{i}\right\|<\infty$. For a fixed function $\varphi \in \mathscr{K}_{m}(V)$, we have

$$
\begin{aligned}
\limsup _{k, n \rightarrow \infty} & \sum_{k<i<n}\left\|L_{i} \varphi^{i-1}(x)\right\| \\
\leqslant & \limsup _{k, n \rightarrow \infty} \sum_{k<i<n}\left\|L_{i}\left(\varphi^{i-1}(x)-x\right)\right\| \\
& +\limsup _{k, n \rightarrow \infty} \sum_{k<i<n}\left\|L_{i}\right\| \cdot\|x\| \\
\quad \leqslant & \limsup _{k, n \rightarrow \infty} \sum_{i=k+1}^{m+1} \frac{(1+m)^{i-1}-1}{m}\left\|L_{i}\right\|\|\varphi-\mathrm{id}\|_{\text {sup }} .
\end{aligned}
$$

By the definition of $W(m)$, we obtain that the sequence $\left\{\frac{(1+m)^{i-1}-1}{m}\left\|L_{i}\right\|\right\}_{i=1}^{\infty}$ is convergent, so

$$
\limsup _{k, n \rightarrow \infty} \sum_{i=k+1}^{m+1} \frac{(1+m)^{i-1}-1}{m}\left\|L_{i}\right\|\|\varphi-\mathrm{id}\|_{\text {sup }}=0,
$$

which means that $\mathscr{P}(\varphi)$ is a well-defined function.
For $\varphi, \psi \in \mathscr{K}_{m}(V)$, by (6) we get

$$
\|\mathscr{P} \varphi-\mathscr{P} \psi\|_{\text {sup }} \leqslant \frac{W(m)}{m}\|\varphi-\psi\|_{\text {sup }} .
$$

If $\psi=$ id, then $\mathscr{P} \psi=\sum_{i=1}^{\infty} L_{i} \mathrm{id}^{i}=\mathrm{id}$. Thus, we have $\| \mathscr{P} \varphi-$ id $\left\|_{\text {sup }}=\right\| \mathscr{P} \varphi-\mathscr{P}(\mathrm{id}) \|_{\text {sup }} \leqslant$ $\frac{W(m)}{m}\|\varphi-\mathrm{id}\|_{\text {sup }}=\frac{W(m)}{m} k(\varphi) \leqslant W(m)$, and consequently that $\mathscr{P} \varphi \in \mathscr{K}(V)$.

By applying Lemma 2 , we have $k\left(\varphi^{i-1}\right) \leqslant(1+$ $k(\varphi))^{i-1}-1$. As $\varphi \in \mathscr{K}_{m}(V)$, we obtain that $k(\varphi) \leqslant$ $m$, then we have $k\left(\varphi^{i-1}\right) \leqslant(1+k(\varphi))^{i-1}-1 \leqslant(1+$ $m)^{i-1}-1$. For any two points $x, y \in V$, we obtain that

$$
\begin{aligned}
& \|(\mathscr{P} \varphi(x)-x)-(\mathscr{P} \varphi(y)-y)\| \\
& \quad \leqslant \sum_{i=1}^{\infty}\left\|L_{i}\right\|\left\|\left(\varphi^{i-1}(x)-x\right)-\left(\varphi^{i-1}(y)-y\right)\right\| \\
& \quad \leqslant \sum_{i=1}^{\infty}\left\|L_{i}\right\| k\left(\varphi^{i-1}\right)\|x-y\| \\
& \quad \leqslant \sum_{i=1}^{\infty}\left\|L_{i}\right\|\left((1+m)^{i-1}-1\right)\|x-y\| \\
& \quad \leqslant W(m)\|x-y\|,
\end{aligned}
$$

and therefore

$$
k_{\mathscr{P}} \leqslant W(m)
$$

Also we have

$$
\begin{aligned}
\operatorname{Lip}(\mathscr{P}) & \leqslant \frac{W(m)}{m}<\frac{m-k(F)}{m(1+m)} \leqslant \frac{m+m k(F)}{m(1+m)} \\
& =\frac{1+k(F)}{1+m} \leqslant 1-W(m) \leqslant 1-k_{\mathscr{P}} .
\end{aligned}
$$

So we have

$$
\frac{1}{1-k_{\mathscr{P}}-\operatorname{Lip}(\mathscr{P})} \leqslant \frac{m}{m-W(m)-m W(m)} .
$$

By Corollary 1, we get the conclusion.

Example 1 Consider the following iterated equation

$$
L_{1}\left(f^{2}(x)\right)+L_{2}(f(x))=F(x) \text { for } x \in V,
$$

where $L_{1}, L_{2} \in \mathscr{L}(X)$, and $L_{1}+L_{2}=\mathrm{id}$. Assume that $\frac{1}{2}<M=\left\|L_{1}\right\|<1$, and take $m=\frac{1}{2}\left(\frac{1}{M}-1\right)<1$. If $k(F)<\frac{1}{4}(1-M)^{2}$, then $W(m)=m\left\|L_{1}\right\|$ and

$$
\begin{aligned}
(1+m)(1-W(m)) & =(1+m)\left(1-m\left\|L_{1}\right\|\right) \\
& =1+m-m(1+m)\left\|L_{1}\right\| \\
& =1+m(1-(1+m) M) \\
& =1+\frac{1}{4}(1-M)^{2}>1+k(F) .
\end{aligned}
$$

So (7) has Hyers-Ulam stability. 
Acknowledgements: The research was supported by National Natural Science Foundation of China (Grant No. 11101105).

\section{REFERENCES}

1. Ulam SM (1964) Problems in Modern Mathematics, Chapter VI, Science Edn, Wiley, New York.

2. Hyers DH (1941) On the stability of the linear functional equations. Proc Natl Acad Sci USA 27, 222-224.

3. Kuczma M, Choczewski B, Ger R (1990) Iterative Functional Equations, Ecyclopedia of Mathematics and its Application, Cambridge University Press, Cambridge.

4. Zhang JZ, Yang L, Zhang WN (1998) Iterative Equations and Embedding Flow, Shanghai Scientific and Technological Education Publishing House, Shanghai.

5. Xu B, Zhang WN (2007) Decreasing solutions and convex solutions of the polynomial-like iterative equation. J Math Anal Appl 329, 483-497.
6. Fontich E (1990) Transversal homoclinic points of a class of conservative diffeomorphisms. J Differ Equ 87, 1-27.

7. Bessis D, Marmi S, Turchetti G (1989) On the singularities of divergent majorant series arising from normal form theory. Rend Math Ser VII 9, 645-659.

8. Greenfield SJ, Nussbaum RD (2001) Dynamics of a quadratic map in two complex variables. $J$ Differ Equ 169, 57-141.

9. Ng CT, Zhang WN (1997) Invariant curves for planar mappings. J Differ Equ Appl 3, 147-168.

10. Zhang WN (1987) Discussion on the iterated equation $\sum_{i=1}^{n} \lambda_{i} f^{i}(x)=F(x)$. Chin Sci Bull 32, 1444-1451.

11. Kulczycki M, Tabor J (2002) Iterative functional equations in the class of Lipschitz functions. Aequationes Math 64, 24-32.

12. Tabor J, Zołdak M (2004) Iterative equations in Banach spaces. J Math Anal Appl 299, 651-662.

13. Xia C, Wang X (2020) Hyers-Uam stability for $C^{1}$ solution of sereies-like iterative equation with variable coefficients. ScienceAsia 46, 240-244. 